\title{
Analysis of expression profiling data suggests explanation for difficulties in finding biomarkers for nasal polyps*
}

\author{
Eun Jung Lee ${ }^{1,2+}$, Danuta R. Gawel', Sandra Lilja', Xinxiu Li', \\ Samuel Schäfer', Oleg Sysoev³ ${ }^{3}$ Huan Zhang', Mikael Benson ${ }^{1,4,5 t}$ \\ 'Centre for Personalized Medicine, Linköping University, Linköping, Sweden \\ 2 Department of Otorhinolaryngology, Yonsei University Wonju College of Medicine, Wonju, Korea \\ ${ }^{3}$ Division of Statistics and Machine Learning, Department of Computer and Information Science, Linköping University, Sweden \\ ${ }^{4}$ Crown princess Victoria Children's Hospital, Linköping University Hospital, Sweden \\ ${ }^{5}$ Wallenberg Centre for Molecular Medicine, Linköping University, Sweden
}

Rhinology 58: 4, 360 - 367, 2020

https://doi.org/10.4193/Rhin19.407

*Received for publication:

November 7, 2019

Accepted: February 12, 2020

${ }^{\dagger}$ These contributed equally as corresponding author

\begin{abstract}
Background: Identification of clinically useful biomarkers for Nasal Polyposis in chronic rhinosinusitis (CRSwNP) has proven difficult. We analyzed gene expression profiling data to find explanations for this.

Methods: We analyzed mRNA expression profiling data, GSE36830, of six uncinate tissues from healthy controls and six NP from CRSwNP patients. We performed Ingenuity Pathway Analysis (IPA) of differentially expressed genes to identify pathways and predicted upstream regulators.
\end{abstract}

Results: We identified 1,608 differentially expressed genes and 177 significant pathways, of which Th1 and Th2 activation pathway and leukocyte extravasation signaling were most significant. We identified 75 upstream regulators whose activity was predicted to be upregulated. These included regulators of known pathogenic and therapeutic relevance, like IL-4. However, only seven of the 75 regulators were actually differentially expressed in NP, namely CSF1, TYROBP, CCL2, CCL11, SELP, ADORA3, ICAM1. Interestingly, these did not include IL-4, and four of the seven were receptors. This suggested a potential explanation for the discrepancy between the predicted and observed expression levels of the regulators, namely that the receptors, and not their ligands, were upregulated. Indeed, we found that 10 receptors of key predicted upstream regulators were upregulated, including IL4R.

Conclusion: Our findings indicate that the difficulties in finding specific biomarkers for CRSWNP depend on the complex underlying mechanisms, which include multiple pathways and regulators, each of which may be subdivided into multiple components such as ligands, soluble and membrane-bound receptors. This suggests that combinations of biomarkers may be needed for CRSwNP diagnostics.

Key words: biomarker, microarray, nasal polyp, pathway, receptor

\section{Introduction}

Chronic rhinosinusitis with nasal polyps (CRSwNP) is one of the most common chronic diseases in adults, which is characterized by fleshy swellings (nasal polyps) that develop in the lining of the nose and paranasal sinuses ${ }^{(1,2)}$. CRSwNP can be accompanied by allergic rhinitis, chronic rhinitis, asthma, gastroesophageal reflux disease, and sleep apnea ${ }^{(3,4)}$.

CRS is defined by the presence of at least two out of four car- dinal symptoms (i.e., facial pain/pressure, hyposmia/anosmia, nasal drainage, and nasal obstruction) for at least 12 consecutive weeks, in addition to objective evidence confirmed by either nasal endoscopy or a computed tomography scan according to the European Position Paper on Rhinosinusitis and Nasal Polyps guideline ${ }^{(1,2)}$. Furthermore, clinical treatment strategies for patients with CRSWNP are limited, in part because the underlying mechanisms contributing to disease pathology are hetero- 
geneous and not entirely known ${ }^{(5,7)}$. Although the exact cause or causes are unknown, decades of painstaking research have led to increasingly detailed understanding of various disease mechanisms, which are found not only in immune cells, but also many other cell types ${ }^{(8-10)}$. This has led to the identification of proteins that are both potential biomarkers for CRSwNP, as well as new therapeutic targets ${ }^{(11)}$. However, clinical implementation of such biomarkers and drugs have proven great challenges. One reason could be the complexity of the underlying mechanisms, which makes prioritization of relevant biomarkers and targets difficult. Another reason may considerable variability of the levels of the identified proteins. As an example, IL4 may have both diagnostic and therapeutic relevance ${ }^{(12)}$. However, its diagnostic value is complicated by low and variable expression levels. A similar complexity is found in many other common diseases. This has spurred unbiased searches using genetics ${ }^{(13,14)}$ transcriptomics ${ }^{(15,16)}$ or proteomics ${ }^{(17)}$. An important experience from such studies is that prioritization of biomarkers requires systems level analyses aiming at overall understanding of disease mechanisms. A key rationale behind such analyses is to identify higher order representations of the complex changes, such as pathways or predicted upstream regulators ${ }^{(18)}$. As examples, the Th2 pathway and IL-4 would be likely representations in CRSwNP. Another experience from previous multi-omics studies is that combinations of biomarkers may be needed to represent the complex mechanisms. Based on these experiences we hypothesized that novel, and potentially more specific, biomarkers for CRSwNP could be identified by network analysis of existing profiling data from cells in NP tissue from CRSWNP patients compared to uncinate process from healthy controls. In order to get a functional overview of the complex gene expression changes, we focused on pathways and upstream regulators rather than individual genes ${ }^{(19-21)}$. In summary, we found 1,608 genes that differed in expression between CRSwNP and uncinate tissue from healthy based on expression profiling data (22). Further analyses led to the identification of a wide variety of canonical pathways and predicted upstream regulators. This complexity could explain the difficulties in finding representative biomarkers. Another explanation was suggested by the analyses of upstream regulators. Among 75 predicted activated upstream regulators, only seven were differentially expressed. Those seven did not include IL4. Since IL4 expression is highly variable, and therapeutic targeting of the membrane-bound IL4 receptor may be effective in CRSWNP, we examined the expression levels of the membrane receptors of IL- 4 and the other predicted activated receptors. We found that the expression levels of the receptors of IL4 and many other predicted activated regulators increased significantly. Thus, increased expression of membrane-bound receptors, rather than their ligands may explain increased activation of downstream pathways.

Taken together, our analyses indicated highly complex pathoge- nic mechanisms in CRSWNP, of which the most significant agreed with previous studies. That complexity may be one explanation for finding biomarkers. Another explanation could be that membrane-receptors rather than their soluble ligands change in expression.

\section{Material and methods}

Identification and selection of eligible gene expression datasets for meta-analysis

We systematically mined the Gene Expression Omnibus (GEO) database for expression profiling datasets (October 2019) ${ }^{(23)}$. The following key words and their combinations were used: "chronic rhinosinusitis, nasal polyp, human, microarray, gene expression dataset". The following information was extracted from each identified study: GEO accession number, sample type, platform, number of cases and controls, references, and gene expression data. We found one proper expression profiling data (GSE36830) that collected uncinate tissues from control subjects and NP tissues from patients with CRSwNP ${ }^{(22)}$. Gene expression profiles were evaluated using Human Genome U133 plus 2.0 array (Affymetrix). Inclusion criteria were set and strictly followed for dataset selection: human case/control study, comparable conditions, untreated samples and availability of raw and processed data.

\section{Analysis of gene expression data}

GSE36830 collected uncinate tissues from 6 control subjects and NP tissues from 6 patients with CRSwNP. Using GEO2R, we identified differentially expressed genes (DEGs) between NP from patients with CRSwNP versus uncinate tissues from healthy control subjects ${ }^{(24,25)}$. The data was annotated using the NCBI generated platform and adjusted for multiple testing using Benjamini-Hochberg procedure. The data was then sorted only to include significant DEGs (FDR < 0.05) for downstream analysis.

\section{Ingenuity pathway analysis}

Briefly, our bioinformatics strategy was based on finding pathways among the DEGs, and upstream regulators of the DEGs. The objective of pathway analysis is the obtain an overview of disease-associated mechanisms, while the objective of upstream regulators is to find key regulators of such mechanisms. Note that each regulator may affect multiple pathways, which often interact in networks. The analyses were performed using the Ingenuity Pathway Analysis (IPA) software (Qiagen, Hilden, Germany, https://www.qiagenbioinformatics.com/products/ ingenuity-pathway-analysis/) (26).

IPA relies on a global database called Ingenuity Knowledge Base (IKB) that contains information about known cause-effect relationships based on manual curation of a vast body of medical literature and biomedical databases, which is continuously updated ${ }^{(27)}$. This database can be represented as a graph that contains tens of thousands of nodes and more than a million of 
A

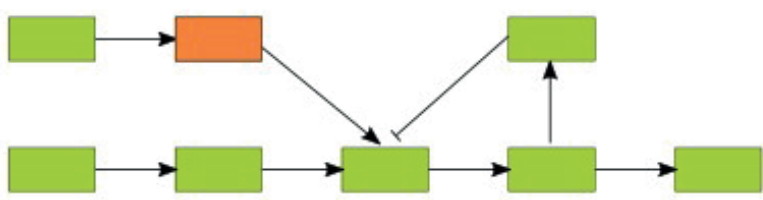

B

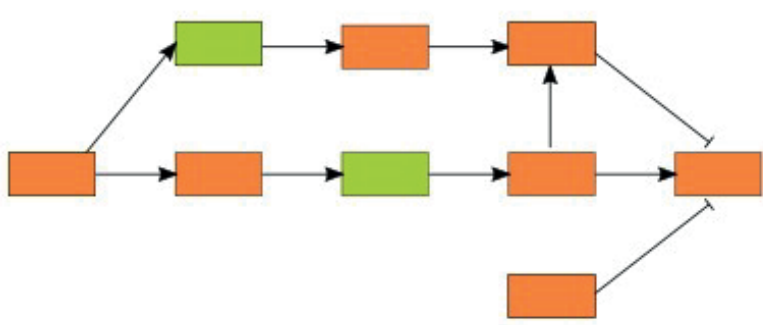

Figure 1. Toy models of two pathways to show the idea behind pathway enrichment of differentially expressed genes. In the upper pathway (A) only one gene is differentially expressed (orange box), while most genes are differentially expressed in the lower pathway (B). Thus, the lower pathway (B) is significantly enriched for differentially expressed genes, and potentially disease-associated.

edges where nodes represent genes, chemicals, protein families etc., and edges represent cause-effect relationships. In order to identify significant pathways, we used the IKB database as a template for statistical comparisons with the DEGs obtained from the input data. If a significant proportion of genes in a pathway is found to be differentially expressed, then that pathway is significant. More specifically, the right-tailed Fisher Exact Test was performed in order to assess whether the association between DEGs and a given pathway is not due to the random chance. This test is based on the number of DEGs and the total number of genes that are known to be associated with a specific pathway. The greater number of DEGs involved in the pathway the greater is the chance that the association is not random. On the other hand, the greater number of genes involved in the pathway the greater is the likelihood that the association might be due to the random chance. Over-represented pathways are those where more DEGs overlap with genes involved in the pathway than expected by chance ( $p$-value $<$ 0.05 ) (Figure 1). To correct for multiple testing, the obtained P-values were adjusted by the Benjamini-Hochberg method. Finally, the pathways associated with the adjusted $p$-values that were lower than a given significance threshold were considered as significant pathways.

The upstream regulators of groups of interacting DEGs were identified by Upstream Regulator Analysis (URA) that is also based on IKB databaset ${ }^{(27)}$. URA requires a set of DEGs as input data set and extracts nodes (potential upstream regulators) that are directly connected in the IKB graph to one or more genes from the input data set. URA returns two quantities per potential upstream regulator: overlap p-value and activation z-score. We focused on the following regulator categories, namely cytokine, growth factor, G-protein coupled receptor, ligand-dependent nuclear receptor, and transmembrane receptor. The overlap $p$-value is obtained by the Fisher's exact test that measures how likely it is that a regulator affects the observed associated amounts of genes by chance. Each edge in the IKB network is associated with the sign: positive or negative, depending on whether the observed effect is activating or inhibiting. At the same time, the DEGs from the input data set are also associated with a sign: positive or negative, depending on the fold change. Z-score aims to compare the sign patterns in order to evaluate consistency between up/down regulation pattern and activation/inhibition pattern. Under assumptions of a random pattern, $\mathrm{Z}$ is normally distributed, and therefore the consistency can be evaluated by checking Z-scores that are large enough.

\section{Results}

Selection of microarray datasets

We searched the GEO database using the terms 'chronic rhinosinusitis', 'nasal polyp' and 'microarray'. This resulted in one mRNA profiling study (GSE36830), of uncinate tissues from control subjects and NP tissues from patients with CRSwNP (22). The study was performed using the Human Genome U133 plus 2.0 array (Affymetrix).

\section{Identification of differentially expressed genes (DEGs) in nasal polyps from CRSwNP}

Comparison of NP tissues from CRSwNP with uncinate tissues from control subjects resulted in 1,608 DEGs (Supplementary material 1). These DEGs included a wide variety of genes, such as fibroblast growth factors, interleukins, mucins like MUC5B, MUC12, MUC19, and chemokine families such as CCL13, CCL8, CCL23. As noted in the discussion below, several of the DEGs agree with current understanding of the pathophysiology of CRSWNP (28).

\section{Identification of pathways in nasal polyps from CRSwNP} In order to gain a functional overview of the complex expression changes in the CRSwNP we performed Ingenuity Pathway Analysis (IPA) to identify disease-associated pathways. This resulted in 177 significant pathways (Supplementary material 2). The top ten pathways (adjusted $p$ value) included Th1 and Th2 activation pathway (6.31E-13), leukocyte extravasation signaling (7.94E-13), Th2 pathway (1.58E-12), CD28 signaling in Thelper cells (1.26E-10), Role of NFAT in regulation of the immune response (2.51E-10), phagosome formation (5.25E-10), granulocyte adhesion and diapedesis (2.40E-09), iCOS-iCOSL signaling in T helper cells (2.51E-09), Natural killer cell signaling (4.57E-09), Th1 pathway (4.57E-09) (Figure 2). These findings agree with activation of T cells being known to be elevated in eosinophilic NPs, as 


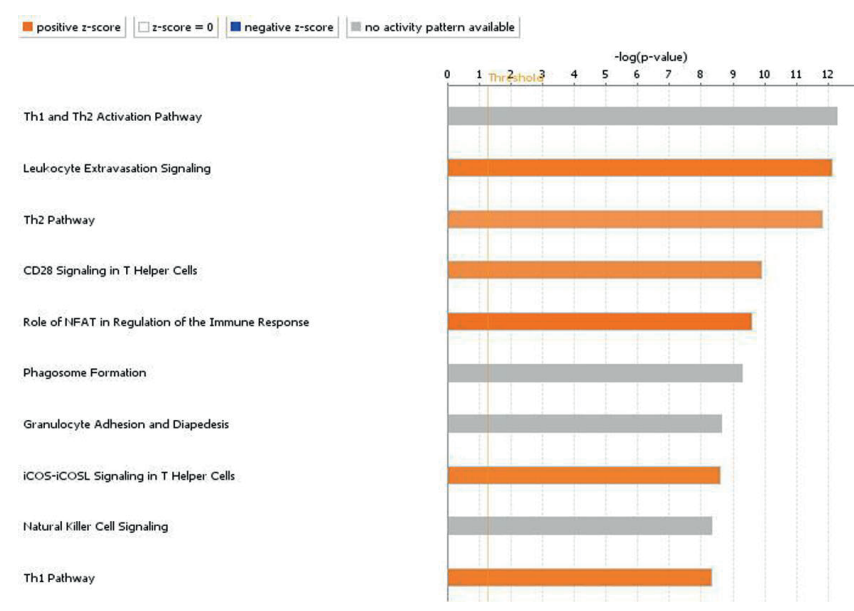

Figure 2. IPA analyses of expression profiling dataset of patients with nasal polyps from chronic rhinosinusitis with nasal polyp compared to uncinate tissues from control subjects. The lengths of the pathway bars indicate significance (-log $\mathrm{p}$ values based on Fisher's exact test righttailed). Orange color indicates activated pathway, and grey that activation or inhibition cannot be inferred based on the expression levels of the genes.

well as T cell accumulation in both mucosal and epithelial areas of NPs ${ }^{(29)}$.

Taken together, the above pathway analyses showed good agreement with previous studies of individual mechanisms. However, collectively the analyses indicate a staggering pathogenic complexity, which makes prioritization of biomarkers a great challenge.

\section{Identification of upstream regulators in nasal polyps from CRSwNP}

Next, we performed IPA analysis of the 1,608 DEGs to find predicted activated upstream regulators of those DEGs. The rationale was that, similar to pathways, such regulators are higher order representations of the complex gene expression changes. We found 75 predicted activated upstream regulators (Supplementary material 3). The top ten upstream regulators among the 75 predicted activated upstream regulators (Z-scores) included TNF (6.003), IFNG (5.702), IL4 (5.506), Vegf (4.861), CSF2 (4.799), IL2 (4.584), HGF (4.527), IL1B (4.047), IL18((3).972), IL1 (3.915) (Table 1, Supplementary material 3).

It is important to note that the upstream regulators were predicted based on their known effects on downstream groups of genes. In other words, if a group of genes showed coordinated changes, potential upstream regulators of those changes were identified, based on previous experimental data accumulated in IPA ${ }^{(26)}$. However, the identified regulators may not necessarily be differentially expressed. We therefore checked if those regulators actually were differentially expressed in CRSWNP. Interestingly, only seven of the 75 predicted activated upstream regulators, CSF1, TYROBP, CCL2, CCL11, SELP, ADORA3, ICAM1,
Table 1. Top 15 predicted activated upstream regulators by IPA analyses of expression profiling dataset of patients with nasal polyps compared to uncinate tissues from control subjects.

\begin{tabular}{lccc}
$\begin{array}{l}\text { Upstream } \\
\text { regulators }\end{array}$ & Molecule Type & $\begin{array}{c}\text { Predicted } \\
\text { status }\end{array}$ & $\begin{array}{c}\text { Activation } \\
\text { Z-score }\end{array}$ \\
\hline TNF & Cytokine & Activated & 6.003 \\
\hline IFNG & Cytokine & Activated & 5.702 \\
\hline IL4 & Cytokine & Activated & 5.506 \\
\hline Vegf & Group & Activated & 4.861 \\
\hline CSF2 & Cytokine & Activated & 4.799 \\
\hline IL2 & Cytokine & Activated & 4.584 \\
\hline HGF & Growth factor & Activated & 4.527 \\
\hline IL1B & Cytokine & Activated & 4.047 \\
\hline IL18 & Cytokine & Activated & 3.972 \\
\hline IL1 & Group & Activated & 3.915 \\
\hline IL5 & Cytokine & Activated & 3.865 \\
\hline EGF & Growth factor & Activated & 3.636 \\
\hline F2R & G-protein coupled receptor & Activated & 3.616 \\
\hline CD40 & Transmembrane receptor & Activated & 3.447 \\
\hline IL27 & Cytokine & Activated & 3.379 \\
\hline
\end{tabular}

were differentially expressed (Table 2). All of these have known regulatory roles in inflammatory responses. Eotaxin 1 (CCL11) has been previously described as elevated in NP. CCL11 is among the main CCR3-activating chemokines responsible for recruitment of eosinophils ${ }^{(30-32)}$. By contrast, Ocampo's unpublished observation by multiplex bead array showed that CCL2 did not differ between NP and control ${ }^{(33)}$. However, it was striking that IL4 was not differentially expressed in NP. This contrasts with previous work of direct diagnostic and therapeutic relevance, in which Bachert, Gevaert and colleagues have shown that antibodies against the IL-4/IL-13 receptor alpha chain shrink NP ${ }^{(12)}$.

Increased receptor expression may explain why predicted upstream regulators are not differentially expressed in nasal polyps from CRSwNP

Since only seven of the 75 predicted activated upstream regulators were differentially expressed, we searched for explanations for this discrepancy. One explanation was suggested by four of the seven differentially expressed regulators being membranebound receptors (Table 2). That explanation would be that if the predicted upstream regulator was a ligand that did not differ between NP and control tissue, the predicted regulator could instead be the receptor of that ligand (if that receptor increased in expression).

Indeed, we found that ten receptors of the 75 predicted upstream regulators were differentially expressed in NP. Those re- 
Table 2. Predicted activated upstream regulators that were differentially expressed in nasal polyps.

\begin{tabular}{|c|c|c|c|c|}
\hline $\begin{array}{l}\text { Upstream } \\
\text { regulators }\end{array}$ & $\begin{array}{l}\text { Expr } \\
\text { Log } \\
\text { Ratio }\end{array}$ & Molecule Type & $\begin{array}{c}\text { Activation } \\
\text { Z-score }\end{array}$ & p-value \\
\hline CSF1 & 0.902 & Cytokine & 2.455 & $1.70 \mathrm{E}-06$ \\
\hline TYROBP & 1.986 & $\begin{array}{c}\text { Transmembrane } \\
\text { receptor }\end{array}$ & 2.433 & $1.02 \mathrm{E}-03$ \\
\hline $\begin{array}{l}\text { CCL2 } \\
(\mathrm{MCP}-1)\end{array}$ & 2.246 & Cytokine & 2.407 & $7.94 \mathrm{E}-03$ \\
\hline $\begin{array}{l}\text { CCL11 } \\
\text { (Eotaxin-1) }\end{array}$ & 2.803 & Cytokine & 2.394 & $5.41 \mathrm{E}-05$ \\
\hline SELP & 1.516 & $\begin{array}{c}\text { Transmembrane } \\
\text { receptor }\end{array}$ & 2.236 & $1.89 \mathrm{E}-02$ \\
\hline ADORA3 & 2.113 & $\begin{array}{l}\text { G-protein coupled } \\
\text { receptor }\end{array}$ & 2 & $1.87 \mathrm{E}-02$ \\
\hline ICAM1 & 1.182 & $\begin{array}{c}\text { Transmembrane } \\
\text { receptor }\end{array}$ & 2 & $2.26 \mathrm{E}-02$ \\
\hline
\end{tabular}

ceptors included CSF1R, CSF2RA, CSF2RB, IL1RL1, IL10RA, IL12RB1, IL18R1, IL2RA, IL2RG, IL27RA, IL3RA and IL4R, whose ligands were both known to be of pathogenic importance and predicted to be upregulated (Figure 3). Therefore, increased receptor expression could be an explanation for altered expression of genes induced by regulators whose expression were not.

\section{Discussion}

CRSwNP is a common disease that causes significant suffering. Diagnosis is complicated by unspecific symptoms, and the need for investigations that may be difficult to perform outside of specialized centres ${ }^{(1-4)}$. Although promising biomarkers have been proposed, these have, in general, not reached the clinic. Treatment options are also limited. Both the diagnostic and therapeutic problems have a common background, namely the complexity of the underlying disease mechanisms ${ }^{(5-11)}$. Gene profiling technology can simultaneously obtain an overview of all such mechanisms by comparing normal and diseased tissues ${ }^{(34,37)}$. In the literature, profiling studies of CRSwNPs and other inflammatory diseases have shown altered expression of thousands of genes ${ }^{(22,34-37)}$. In this study, we aimed to find explanations for the difficulties in finding biomarkers by a strategy focusing on identifying higher order representations of the complex expression changes in CRSwNP.

Our analyses showed 177 significant pathways, of which the Th1 and Th2 activation pathway was most significant. This agrees with the understanding that this pathway and its upstream regulators, IL-4 and IL-13, may have both diagnostic and therapeutic implications. Unpublished studies by Sanofi/Regeneron (multiple press releases are available) indicate that dupilumab, an antibody against the alpha chain of the IL-4 and IL-13 receptors, has impressive beneficial effects in nasal polypoid lesions ${ }^{(33)}$.

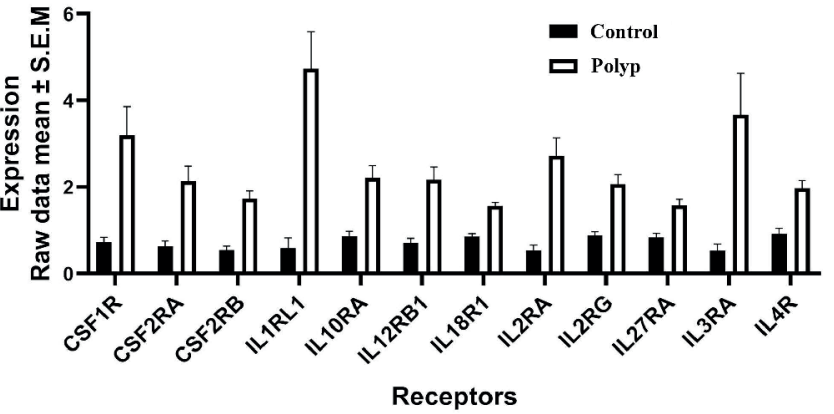

Figure 3. Expression levels of the receptors of predicted upstream regulators in nasal polyps (white bars) compared to controls (black bars). The receptors ( $p$ values) were: CSF1R (0.018), CSF2RA (0.034), CSF2RB (0.011), IL1RL1 (0.024), IL10RA (0.026), IL12RB1 (0.02), IL18R1 (0.023), IL2RA (0.015), IL2RG (0.027), IL27RA (0.043), IL3RA (0.034), IL4R (0.031).

Moreover, the expression of CCL11 by NP-derived fibroblasts, or airway epithelial cells, has been shown to be increased by the combination of IL-4, or IL-13, as well as TNF in vitro, suggesting that there may be a positive feedback loop for eosinophil recruitment that is further enhanced in a pro-inflammatory type 2 inflammatory environment ${ }^{(38)}$. However, in our analyses it was striking that IL4 was not differentially expressed in CRSwNP. Similarly, we noted that four out of seven predicted upstream regulators that were differentially expressed, were receptors. Importantly, these included IL4R. Thus, a potential explanation for lack of reliable soluble biomarkers, like IL4, could be increased expression of receptors, rather than their soluble ligands. Indeed, we found that ten receptors to 75 predicted upstream regulators were actually differentially expressed. Those receptors included CSF1R, CSF2RA, CSF2RB, IL1RL1, IL10RA, IL12RB1, IL18R1, IL2RA, IL2RG, IL27RA, IL3RA, and IL4R. As previously discussed by us and others, such receptors may have both inhibitory and activating roles, depending on their mechanisms of action, as well as their levels relative to their ligand and membranebound receptors ${ }^{(39)}$. Thus, diagnostic analyses of any predicted regulator may need to take into account the relative expression levels of the corresponding levels of the cognate soluble and membrane-bound receptors.

On top of this complexity, our further analyses implicated a great variety of genes, whose protein products could have diagnostic relevance. These included fibroblast growth factors, interleukins, mucins such as MUC5B, MUC12, MUC19, and chemokine families such as CCL13, CCL8, CCL23. These findings agree with current understanding of the pathophysiology of CRSwNP (28). For example, mucus hypersecretion is one of the main symptoms and can occur as a result of an increased mucus production by serous cells of the submucosal glands in the airways. Mahmoud et al. previously showed complex patterns of expression of a large set of mucin genes in NP ${ }^{(40)}$. Differential expression of chemokines agrees with overproduction of eosinop- 
hil-promoting $\mathrm{C}-\mathrm{C}$ chemokines by the sinus epithelium, perhaps driven in part through innate stimuli, as well as TH2 cytokines, such as IL-13 ${ }^{(28)}$. Our pathway analyses also coincided with other studies ${ }^{(41-43)}$. For example, Ma et al. showed that dramatically enhanced nuclear factor of activated T cells (NFAT) localization was observed in NP compared to controls ${ }^{(42)}$, which agrees with our data showing role of NFAT in regulation of the immune response in CRSwNP. Rigas et al. showed blocking of ICOS-ICOSL interaction with anti-ICOSL reduced airway hyperresponsiveness (AHR) and eosinophilia ${ }^{(43)}$. This reflects that iCOS-iCOSL signaling in Thelper cells pathway can be increased in airway inflammatory diseases such as NP. The above genes and mechanisms are part of a complex interplay between muco-ciliary clearance, epithelial barrier dysfunction, the host immune response, and tissue remodeling, all of which are thought to work in concert and contribute to the chronic inflammation characteristic of CRSwNP (6).

Yao et al. suggested that TYROBP could be a potential important biomarker for CRSWNP ${ }^{(44)}$. We also found TYROBP was differentially expressed in CRSwNP as well as acted as predicted activated upstream regulator. Seshadri et al. showed that decreased SPLUNC1 and LPLUNC2 in NPs reflects a profound decrease in the number of submucosal glands leading to a release of glandular innate defense molecules ${ }^{(22)}$. Furthermore, Stevens et al. used principal component analysis (PCA) to aid in the interpretation of the entire multivariate immunoplex data and showed that NP samples, as a whole, had higher expression of eotaxin-1, eotaxin-2, eotaxin-3, MCP-1, MCP-4, TARC, IL-5, IL-10, and IL-13 relative to controls, which coincide with our top upstream regulators in CRSwNP (45).

Limitations of this study include that only one material was analyzed, and that mRNA levels may not necessarily correspond to protein levels. On the other hand, our findings are supported by previous studies of individual mechanisms. CRSwNP is a heterogeneous disease entity that includes different endotypes such as CRS with and without asthma, polyps that do or do not recur, as well as with or without eosinophil/neutrophil enrichment. However, we present a systems-level overview of pathways and upstream regulators, which indicates the complexity of CRSwNP pathogenesis, and potentially also the relative importance of the identified mechanisms.

Given that The European Position Papers on Rhinosinusitis from 2005, 2007 and 2012 have highlighted the impact of CRSwNP on quality of life, there is a considerable need for systems-level understanding of CRSwNP in order to find biomarkers and therapeutic targets ${ }^{(1,2,46)}$. Indeed, new and expanded areas of research for EPOS2020 included gene profiling technology as well as precision medicine in rhinology ${ }^{(46)}$. As an example, treatment of refractory CRSwNP is an important future research direction.
These result in great costs and suffering ${ }^{(47)}$. Refractory CRSwNP also indicate the complexity and heterogeneity of CRSWNP. Refractory CRSwNP, which is associated with eosinophilia, asthma, allergy, and deficiencies in muco-ciliary dysfunction appear to be secondary to an interaction of a susceptible host with the outside environment ${ }^{(48)}$. According to EPOS criteria for CRS treatment, biologicals, such as treatment targeting the IL4 receptor (IL4R), may be considered if first line treatments are ineffective ${ }^{(12)}$. However, treatment effects of biologicals also vary. Our results point to one potential explanation, namely variable expression IL4R and other receptors. We propose that our data may be used for hypothesis-based studies of mediators and receptors in refractory polyps. Further studies of such receptors in responders and non-responders to biologicals are warranted.

\section{Conclusion}

In summary, our findings indicate that the difficulties in finding specific biomarkers for CRSwNP depend on the complex underlying mechanisms, which include multiple pathways and regulators, each of which may be subdivided into multiple components such as ligands, soluble and membrane-bound receptors. Thus, combinations of biomarkers may be needed for CRSwNP diagnostics.

\section{Acknowledgements}

This work was supported by the Swedish Cancer Foundation grants 170542 and 15 0532, European Commission grant 305033, East Gothia Regional Funding, the Swedish Research Council grants 2015-02575, 2015-03495, and 2015-03807, Clinical Cancer Research, Jönköping Sweden, Torsten Söderberg Foundation.

\section{Authorship contribution}

DRG, SL, and SS managed data extraction and bioinformatics analysis. $\mathrm{XL}$ and $\mathrm{HZ}$ interpreted data and revised manuscript. OS contributed to statistical analyses. EJL and MB did IPA and wrote the manuscript. EJL and MB initiated and supervised the study. All authors read and approved of the final manuscript.

\section{Consent to publish}

Not applicable.

\section{Conflict of interest}

Authors declare that they have no competing financial interests.

\section{Ethics approval and consent to participate}

This study was conducted in accordance with the Declaration of Helsinki. 


\section{References}

1. Fokkens W, Lund V, Mullol J. European Position Paper on Rhinosinusitis and Nasa Polyps group. European position paper on rhinosinusitis and nasal polyps 2007. Rhinol Suppl. 2007; 20: 1-136.

2. Fokkens WJ, Lund VJ, Mullol J, et al. European Position Paper on Rhinosinusitis and Nasal Polyps 2012. Rhinol Suppl. 2012; 23: 3 p preceding table of contents, 1-298.

3. Grigoreas C, Vourdas D, Petalas K, Simeonidis G, Demeroutis I, Tsioulos T. Nasal polyps in patients with rhinitis and asthma. Allergy Asthma Proc. 2002; 23(3): 169-174.

4. Tan BK, Chandra RK, Pollak J, et al. Incidence and associated premorbid diagnoses of patients with chronic rhinosinusitis. J Allergy Clin Immunol. 2013; 131(5): 13501360.

5. Dykewicz MS. Rhinitis and sinusitis. Allergy Clin Immunol. 2003; 111 (2 Suppl): S520-S529.

6. Stevens WW, Lee RJ, Schleimer RP, Cohen NA. Chronic rhinosinusitis pathogenesis. J Allergy Clin Immunol. 2015; 136(6): 14421453

7. Lee S, Lane AP. Chronic rhinosinusitis as a multifactorial inflammatory disorder. Curr Infect Dis Rep. 2011; 13(2): 159-168.

8. Halderman A, Lane AP. Genetic and immune dysregulation in chronic rhinosinusitis. Otolaryngol Clin North Am. 2017 50(1): 13-28.

9. Wang H, Pan L, Liu Z. Neutrophils as a protagonist and target in chronic rhinosinusitis. Clin Exp Otorhinolaryngol. 2019; 12(4) 337-347.

10. Brescia G, Sfriso P, Marioni G. Role of blood inflammatory cells in chronic rhinosinusitis with nasal polyps. Acta Otolaryngol. 2019; 139(1): 48-51.

11. Agarwal A, Spath D, Sherris DA, Kita $H$ Ponikau JU. Therapeutic antibodies for nasal polyposis treatment: where are we headed? Clin Rev Allergy Immunol. 2019 May 10 [Epub ahead of print]

12. Bachert C, Mannent $L$, Naclerio RM, et al. Effect of subcutaneous dupilumab on nasal polyp burden in patients with chronic sinusitis and nasal polyposis: a randomized clinical trial. JAMA. 2016; 315(5): 469-479.

13. Bohman A, Juodakis J, Oscarsson M, Bacelis J, Bende M, Torinsson Naluai Å. A familybased genome-wide association study of chronic rhinosinusitis with nasal polyps implicates several genes in the disease pathogenesis. PLoS One. 2017; 12(12) e0185244.

14. Hsu J, Avila PC, Kern RC, Hayes MG, Schleimer RP, Pinto JM. Genetics of chronic rhinosinusitis: state of the field and directions forward. J Allergy Clin Immunol. 2013; 131(4): 977-993, 993.e1-5.

15. Peng $Y, Z i X X$, Teng TF, et al. Wholetranscriptome sequencing reveals heightened inflammation and defective hostdefense responses in chronic rhinosinusitis with nasal polyps. Eur Respir J. 2019. 54 (5)
1900732.

16. Wang W, Gao Z, Wang H, Li T, He W, LV W, Zhang J. Transcriptome analysis reveals distinct gene expression profiles in eosinophilic and noneosinophilic chronic rhinosinusitis with nasal polyps. Sci Rep. 2016; 6: 26604

17. Kim YS, Han D, Kim J, et al. In-depth, proteomic analysis of nasal secretions from patients with chronic rhinosinusitis and nasal polyps. Allergy Asthma Immunol Res. 2019; 11(5): 691-708.

18. Gawel DR, Serra-Musach J, Lilja S, et al. A validated single-cell-based strategy to identify diagnostic and therapeutic targets in complex diseases. Genome Med. 2019; 11(1): 47

19. Benson M, Carlsson B, Carlsson LM, Mostad P, Svensson PA, Cardell LO. DNA microarray analysis of transforming growth factor-beta and related transcripts in nasal biopsies from patients with allergic rhinitis. Cytokine. 2002; 18(1): 20-25

20. Barrenäs F, Andersson B, Cardell LO, et al Gender differences in inflammatory proteins and pathways in seasonal allergic rhinitis. Cytokine. 2008; 42(3): 325-329.

21. Bruhn S, Fang Y, Barrenäs F, et al. A generally applicable translational strategy identifies S100A4 as a candidate gene in allergy. Sci Transl Med. 2014; 6(218): 218ra4.

22. Seshadri S, Lin DC, Rosati M, et al. Reduced expression of antimicrobial PLUNC proteins in nasal polyp tissues of patients with chronic rhinosinusitis. Allergy. 2012; 67(7): 920-928.

23. National Center for Biotechnology Information, Home - GEO - NCBI, (2015). https://www.ncbi.nlm.nih.gov/geo/ (accessed Oct 31, 2019).

24. Smyth GK. Linear models and empirical bayes methods for assessing differential expression in microarray experiments, Stat Appl Genet Mol Biol. 2004; 3: Article3.

25. NCBI, About GEO2R - GEO - NCBI, (n.d.). https://www.ncbi.nlm.nih.gov/geo/info/ geo2r.html (accessed Oct 31, 2019).

26. QIAGEN Inc., https://www.qiagenbioinformatics.com/products/ingenuity-pathwayanalysis/ (accessed Oct 31, 2019).

27. Krämer A, Green J, Pollard J Jr, Tugendreich S. Causal analysis approaches in ingenuity pathway analysis. Bioinformatics. 2014; 30(4): 523-530.

28. Hamilos DL. Drivers of chronic rhinosinusitis: Inflammation versus infection. J Allergy Clin Immunol. 2015; 136(6): 1454-1459.

29. Kato A. Immunopathology of chronic rhinosinusitis. Allergol Int. 2015; 64(2): 121-130.

30. Bartels J, Maune S, Meyer JE, Kulke R, Schlüter C, Röwert J, Christophers E, Schröder JM. Increased eotaxin-mRNA expression in non-atopic and atopic nasal polyps: comparison to RANTES and MCP-3 expression. Rhinology. 1997; 35(4): 171-174.

31. Bachert C, Gevaert P, Holtappels G, Johansson SG, van Cauwenberge P. Total and specific lgE in nasal polyps is related to local eosinophilic inflammation. J Allergy
Clin Immunol. 2001; 107(4): 607-614.

32. Jahnsen FL, Haye R, Gran E, Brandtzaeg P, Johansen FE. Glucocorticosteroids inhibit mRNA expression for eotaxin, eotaxin-2, and monocyte-chemotactic protein-4 in human airway inflammation with eosinophilia. J Immunol. 1999; 163(3): 1545-1551.

33. Hulse KE, Stevens WW, Tan BK, Schleimer RP. Pathogenesis of nasal polyposis. Clin Exp Allergy. 2015; 45(2): 328-346.

34. Lee JY, Lee $S H$, Lee HM, Lee $S H$, Jung HH, Lee SW, Kang SM. Analysis of gene expression profiles of normal human nasal mucosa and nasal polyp tissues by SAGE. J Allergy Clin Immunol. 2006; 118(1): 134-142.

35. Ninomiya T, Noguchi E, Haruna $T$, et al. Periostin as a novel biomarker for postoperative recurrence of chronic rhinosinitis with nasal polyps. Sci Rep. 2018; 8(1): 11450.

36. Liu Z, Kim J, Sypek JP, Wang IM, Horton H, Oppenheim FG, Bochner BS. Gene expression profiles in human nasal polyp tissues studied by means of DNA microarray. J Allergy Clin Immunol. 2004; 114(4): 783-790.

37. Wang X, Dong Z, Zhu DD, Guan B. Expression profile of immune-associated genes in nasal polyps. Ann Otol Rhinol Laryngol. 2006; 115(6): 450-456.

38. Yoshifuku K, Matsune S, Ohori J, Sagara $Y$ Fukuiwa T, Kurono Y. IL-4 and TNF-alpha increased the secretion of eotaxin from cultured fibroblasts of nasal polyps with eosinophil infiltration. Rhinology. 2007; 45(3): 235-241.

39. Benson M, Wennergren G, Fransson M Cardell LO. Altered levels of the soluble IL-1, $\mathrm{IL}-4$ and TNF receptors, as well as the IL-1 receptor antagonist, in intermittent allergic rhinitis. Int Arch Allergy Immunol. 2004 134(3): 227-232

40. Ali MS, Wilson JA, Bennett M, Pearson JP. Mucin gene expression in nasal polyps. Acta Otolaryngol. 2005; 125(6): 618-624.

41. Ley K, Laudanna C, Cybulsky MI, Nourshargh S. Getting to the site of inflammation: the leukocyte adhesion cascade updated. Nat Rev Immunol. 2007; 7(9): 678-689.

42. Ma Y, Zheng C, Shi L. The kinase LRRK2 is differently expressed in chronic rhinosinusitis with and without nasal polyps. Clin Transl Allergy. 2018; 8: 8 .

43. Rigas D, Lewis G, Aron JL, et al., Type 2 innate lymphoid cell suppression by regulatory $T$ cells attenuates airway hyperreactivity and requires inducible T-cell costimulator-inducible T-cell costimulator ligand interaction. J Allergy Clin Immunol. 2017; 139(5): 1468-1477.

44. Yao Y, Xie S, Wang F. Identification of key genes and pathways in chronic rhinosinusitis with nasal polyps using bioinformatics analysis. Am J Otolaryngol. 2019; 40(2): 191-196.

45. Stevens WW, Ocampo CJ, Berdnikovs S, et al. Cytokines in chronic rhinosinusitis. Role in eosinophilia and aspirin-exacerbated respiratory disease. Am J Respir Crit Care Med. 2015; 192(6): 682-694 
46. Fokkens W, Desrosiers M, Harvey R, et al EPOS2020: development strategy and goals for the latest European Position Paper on Rhinosinusitis. Rhinology. 2019; 57(3): 162168.

47. Rudmik L, Smith TL, Schlosser RJ, Hwang $\mathrm{PH}$, Mace JC, Soler ZM. Productivity costs in patients with refractory chronic rhinosinusitis. Laryngoscope. 2014; 124(9): 2007-2012.

48. Batra PS, Tong L, Citardi MJ. Analysis of comorbidities and objective parameters in refractory chronic rhinosinusitis. Laryngoscope. 2013;123 Suppl 7: S1-S11.

\section{Eun Jung Lee}

Department of Otorhinolaryngology

Yonsei University

Wonju College of Medicine

Ilsan-ro 20

Wonju

Republic of Korea

E-mail: starlej@yonsei.ac.kr
Mikael Benson

The Center for Personalized Medicine, Linköping University

58183 Linköping

Sweden

E-mail: mikael.benson@liu.se

\section{Supplementary Data (available upon request)}

Supplementary material 1. List of differentially expressed genes from expression profiling dataset of nasal polyp from patients with chronic rhinosinusitis versus inferior turbinate tissues from healthy subjects (GSE36830).
Supplementary material 2. List of canonical pathways from expression profiling dataset of nasal polyp from patients with chronic rhinosinusitis versus inferior turbinate tissues from healthy subjects (GSE36830).

Supplementary material 3. List of upstream regulators from expression profiling dataset of nasal polyp from patients with chronic rhinosinusitis versus inferior turbinate tissues from healthy subjects (GSE36830). 